Article

\title{
"It Is Part of Belonging": Walking Groups to Promote Social Health amongst People Living with Dementia
}

\author{
Jane M. Robertson *, Grant Gibson, Catherine Pemble, Rog Harrison, Kim Strachan and Sheila Thorburn \\ Faculty of Social Sciences, University of Stirling, Stirling, FK9 4LA, UK; E-Mails: j.m.robertson@stir.ac.uk (J.M.R.), \\ grant.gibson@stir.ac.uk (G.G.), catherine.pemble@stir.ac.uk (C.P.), rogharrisonglasgow@gmail.com (R.G.), \\ kstrachan2020@gmail.com (K.S.), sheila@icywater.com (S.T.)
}

* Corresponding author

Submitted: 14 January 2020 | Accepted: 29 April 2020 | Published: 31 July 2020

\begin{abstract}
People with dementia often report experiencing a 'shrinking world' connected with reduced opportunities to access physical and social spaces. This article applies the framework of social health (Dröes et al., 2017; Huber et al., 2011) as a theoretical lens through which to consider how inclusive walking groups can facilitate access to places and spaces to support people with dementia to remain connected in their communities. Findings are reported from walking interviews and focus group discussions with people with dementia, family carers, volunteers and walk leaders who participated in a national programme of dementia-friendly walking groups in Scotland. Thematic analysis of the data demonstrates that participation has a positive impact on social health, supporting people living with dementia to fulfil their potential, to engage in meaningful activity and to manage both their condition and their wider lives. Benefits include providing a context for continuing social participation and relationships for people with dementia and family carers. Additionally, groups provide a safe space where people with dementia can walk with autonomy and help to reinforce a sense of capacity and agency. Wider implications include the role of walking groups in fostering interdependencies between people with dementia and their wider communities by promoting an enabling ethos of dementia 'inclusiveness.' The benefits of developing an inclusive and supportive approach to involving people living with dementia in walking groups could extend more broadly to the wider community, with such initiatives acting as a catalyst for growing levels of social participation.
\end{abstract}

\section{Keywords}

dementia; community; environment; inclusion; outdoors; social health; walking

\section{Issue}

This article is part of the issue "Home, Housing and Communities: Foundations for Inclusive Society" edited by Isobel Anderson (University of Stirling, UK), Vikki McCall (University of Stirling, UK) and Joe Finnerty (University College Cork, Ireland).

(C) 2020 by the authors; licensee Cogitatio (Lisbon, Portugal). This article is licensed under a Creative Commons Attribution 4.0 International License (CC BY).

\section{Introduction}

Dementia represents a growing health and social care challenge facing most Western societies, including the UK where 800,000 people currently live with dementia (Prince et al., 2014). Recognising that people with dementia want to be supported in the community, attention is turning towards the question of how people with dementia can 'live well' throughout their lives (Alzheimer's Disease International, 2019). Difficulties associated with cognitive impairment and its social stigma mean people with dementia frequently experience a 'shrinking world' characterised by reduced opportunities to access physical and social spaces (Duggan, Blackman, Martyr, \& van Schaik, 2008). This restriction can lead to a loss of pre-existing social relationships, and limit access to outdoor spaces and to meaningful activities enjoyed prior to dementia's onset (Noone, Innes, Kelly, \& Mayers, 2017). Providing opportunities to sustain social relationships through access to outdoor spaces is, therefore, integral to enabling people to remain as members of their communities as their dementia progresses. 
Dementia policy increasingly frames this aim in terms of 'dementia-friendly' communities; an approach adopted across many countries including the UK (Department of Health, 2015). Dementia-friendly communities, it is argued, value participation, inclusion and accessibility as well as human rights and social inclusion (Bartlett, 2016). In doing so, dementia-friendly communities support a shift in focus from institutional to community-based support. As aspirational as these efforts are, they are not without flaws. Serious concerns, for example, remain around whether these efforts put too great an emphasis on the physical environment, thus failing to address significant social and structural factors that may isolate or exclude people with dementia (Wright, 2014). Such perspectives argue that what is needed is not a community that is dementia 'friendly' but one that is 'dementia-enabling' (Swaffer, 2015). This phrasing posits that the opposite of a 'dementia-friendly' community is not one that is 'unfriendly' but a community that actively disables and inhibits the rights and agency of its residents with dementia (Shakespeare, Zeilig, \& Mittler, 2019).

There is some evidence for this within the literature. For example, Phinney, Kelson, Baumbusch, O'Connor, and Purves' (2016) study of a neighbourhood walking initiative demonstrates how an ethos of 'dementiafriendliness' can lead to circumstances in which initiatives are provided exclusively for people with dementia, with less focus on the interaction between participants and the wider communities in which they are situated. As they argue, this can lead to tensions regarding how dementia may define (or not) those attending such group activities. Such structures, arguably, serve only to introduce a sense of geographic diversity to otherwise rigid 'grey ghettos' (Wild, Clelland, Whitelaw, Fraser, \& Clark, 2018) despite the best intentions of those involved. With such arguments in mind, therefore, there is a requirement for activities that are meaningful, enjoyable and beneficial for people with dementia to be provided in a way that promotes inclusion over isolation, and access over restriction. Walking groups, we argue, have the potential to achieve exactly that, attending to the potential value of outdoor spaces, which have received less attention in the literature compared to the built environment when considering how communities might facilitate participation.

Accessing outdoor spaces as part of a meaningful activity has been identified as a powerful mechanism for enabling people with dementia to remain socially and physically engaged (Ward et al., 2018). Indeed, a growing body of literature demonstrates that these can take many forms, such as tending and cultivating green spaces (Noone et al., 2017), accessing woodland (Gibson, Ramsden, Tomlinson, \& Jones, 2017) or walking (Phinney et al., 2016). In doing so, these activities support the well-documented benefits of being outdoors, which include improved affect, wellbeing, sleep and functional abilities (Blake \& Mitchell, 2016; Gonzalez \& Kirkevold, 2013). These complement the benefits of social engage- ment in meaningful activities, such as improved physical, mental and social health (Genoe, 2010), alongside reduced levels of agitation and a more coherent sense of self (Hendriks, van Vliet, Gerritsen, \& Dröes, 2016). As such, meaningful activities that encourage both outdoor access and socialisation have the capacity to support not only psychological and physical wellbeing, but also the social health of people with dementia.

The concept of 'social health,' put forward by Huber et al. (2011), Dröes et al. (2017) and Vernooij-Dassen and Jeon (2016) is one means of incorporating rights, capabilities and citizenship of people with dementia despite cognitive or functional decline (Dröes et al., 2017). Emerging in part from the application of social models of health and disabilities within dementia studies, social health can be defined as (1) capacity to fulfil one's potential, (2) ability to manage life with some degree of independence, and (3) continued participation in meaningful activities such as work, hobbies or leisure (Dröes et al., 2017; Huber et al., 2011). Such a model goes beyond the social model of health, by recognising that social processes and relations can also facilitate health activities, behaviours and outcomes leading to good health (Yuill, Crinson, \& Duncan, 2010). Vernooij-Dassen and Jeon (2016) argue that the core benefits of the social health paradigm include its recognition of people with dementia's ability to participate in social life and its focus on people's capacities, rather than conceptualising health/illness according to a deficit-based model. The concept of social health itself seeks to acknowledge the potential for personal wellbeing and meaningful engagement with one's needs and interests to occur alongside and in the context of long-term and degenerative health conditions, including dementia.

Indeed, emphasising the importance of social health alongside traditional biomedical concerns serves to highlight the vital role that rights, capabilities and citizenship play in maintaining a person with dementia's broader health (Dröes et al., 2017; Huber et al., 2011; Vernooij-Dassen \& Jeon, 2016). This article therefore investigates the experiences of people with and without dementia who attend a national 'dementia-friendly' walking group initiative. The overarching research question for this project was: What is the impact of attending dementia-friendly walking group initiatives on the social health of attendees (both people living with dementia and their family carers)?

\section{Methods}

\subsection{Methodological Design}

The study adopted a co-produced, participatory methodology, drawing on an existing community research partnership forged between the first three authors, who are academic researchers, and the last three authors, who are trained in qualitative research methods (Greasley-Adams et al., 2017, 2019). This approach seeks 
to distribute power more equitably within research relationships and encourages greater engagement between the research as a conceptual and practical project and the communities involved (Ottmann, Laragy, Allen, \& Feldman, 2011). This approach ensured every member of the research team played a significant role; from research design, collecting and analysing data, to reporting results (Vaughn et al., 2018). Ethical approval for the study was awarded by the General University Ethics Panel at the University of Stirling (GUEP62 and GUEP296).

\subsection{Setting}

"Paths for All" is a Scottish charity that supports National Health Service trusts, local authorities and community organisations to run a range of free walking activities for local people. 'Dementia-friendly' walks represent a small but significant subset of a wider group of freeat-the-point-of-delivery 'health walks' which aim to promote activity amongst people who live with long-term health conditions. To be classed as 'dementia-friendly,' walk leaders must receive dementia-awareness training and cascade that knowledge across walking group volunteers. Projects are also required to review and risk assess their walking routes with a specific focus on the impact a dementia diagnosis can have on an individual's physical abilities, perception and cognition.

At the time of the study, "Paths for All" supported 23 walking groups across Scotland to offer 'dementiafriendly' walks in a range of urban green spaces and rural locations, with six groups selected by the charity group to take part in the study. These walking groups typically met weekly, taking a circular route lasting approximately one hour, with a set end-point usually at a local café. While all six groups sought to be 'dementia-friendly,' in five groups people with dementia and carers attended walks alongside the wider local population within each area. The goal of these groups was to ensure that people with dementia who chose to attend were supported by walk volunteers. Attendance of people with dementia varied in each group, but usually comprised a minority of total attendees, with one to three couples (people with dementia and carers) attending each of the walks attended by researchers. One group was set up exclusively for people with dementia and their carers to attend, with approximately 20 people attending this group. In all cases people with dementia who attended walks were accompanied by an informal carer. One group took place in a rural setting, another in a semi-rural area near water, with the remaining four groups operating in natural spaces within towns and villages. These 'blue' and 'green' spaces provided opportunities for participants to walk in nature within both urban and rural environments.

\subsection{Participants and Methods}

Primary fieldwork took place between April and June 2017 and involved research dyads containing one aca- demic and one community researcher attending six walking groups. Where possible, walking interviews were conducted concurrently with two participants from each group, one person identified as having dementia alongside a carer, friend or relative. A focus group followed each walk, wherein walkers, volunteers and walk leaders were invited to discuss the key facilitators, barriers and benefits of participating in each of the walking groups. Informed consent was collected from all participants using principles of process consent (Dewing, 2007). All people with dementia who took part demonstrated capacity at the time of their involvement. Focus groups also took place in December 2017 with health walk coordinators.

\subsection{Walking Interviews}

Walking interviews were the key method used in this study. The practice of interviewing participants about their experiences of walking with the group while doing so is inherently facilitative, as it allows participants to reflect on their experiences in situ, aiding recall and supporting participation (Ward \& Campbell, 2013). This method allowed the act of walking itself to elicit insights and reflections between participants, as being in and around the group while walking in the local area stimulated memories of their past (Clark \& Emmel, 2010). The presence of naturalistic prompts helped participants identify what was and was not meaningful for them as they reflected on their experiences of attending walking groups, as well as what they liked or did not like about the initiative. As such, walking interviews supported social interaction between interviewers and interviewees (Odzakovic, Hellström, Ward, \& Kullberg, 2018) without limiting the naturalistic interactions between group members or compromising the social nature of the groups themselves.

\subsection{Focus Groups}

At the end of each walk, attendees, volunteers and walk leaders were invited to take part in a focus group to explore experiences of walking among the whole group. These six focus group discussions supplemented walking interviews and provided invaluable insights into the perspectives of walkers more broadly. Separate focus groups were conducted with paid coordinators from health walk projects to explore the practical and pragmatic issues surrounding the delivery and facilitation of 'dementiafriendly' walking groups.

\subsection{Coproduction Methods and Data Analysis}

The first workshop brought together academic and community researchers to develop research questions and interview schedules, while the second focused on data analysis. During initial analysis, academic and community researchers worked in pairs to identify initial codes and themes relating to perceived benefits and expe- 
riences of participating in a 'dementia-friendly' walking group (Braun \& Clarke, 2006). The themes identified were: being with other people; being outdoors; atmosphere, ethos and accessibility; safety and security; leadership and organisation. The academic researchers wrote up the analysis based on these themes, which was reviewed for accuracy by the community researchers. A secondary analysis was conducted by the academic researchers to apply social health as a theoretical lens through which to analyse the data, based on the following two questions:

1. What contribution does a community-based walking group initiative make to the social health of people living with dementia?

2. What are the experiences through which an inclusive 'dementia-friendly' walking group is judged to improve social health?

In the secondary analysis, interview and focus group data were subjected to thematic analysis, using the two questions listed above and informed by the constant comparative method. The first two authors worked together using these research questions to organise the analysis. Grant Gibson searched for initial themes, with Jane $M$. Robertson reviewing these themes and developing them into a thematic map. The authors then worked together to refine these themes, with initial themes organised into an overall analytical framework. Catherine Pemble reviewed the framework and worked with the first two authors to refine the themes further. The identified framework relating to social health comprised three overall themes: being with others; reciprocity and looking out for each other; and promoting agency and capacity. After this stage, quotes from transcripts were identified to support these identified themes, as set out in the findings below.

\section{Findings}

Supporting quotations are differentiated by whether collected in a focus group or during an individual walking interview, with the group the participant attended indicated as FG1-FG6, and FG7 used to indicate the discussion with health walk coordinators.

\subsection{Being with Others: Social Inclusion, Participation and Confidence}

The most prominent benefit discussed by walking group attendees was the increase in their social integration and their social health more broadly. Central to this discussion was the idea that individuals interacted with a more diverse group than might happen elsewhere. For the majority, attending the walks gave people the opportunity to meet and spend time with other people, in a relatively safe, supported environment. Many people with dementia experienced a declining social sphere as a result of their condition, the groups alleviated this trend by providing attendees an opportunity to develop existing and new social relationships despite their dementia.

FG1 Participant: That is why I joined.

Interviewer: For the social bit?

FG1 Participant: Yes, because I am just on my own, usually-I think it is a way of meeting people.

The act of walking in the groups encouraged group members to "speak to different company" (FG6), emphasised by a person with dementia who reported "anybody" could join "no matter if there [was] nothing wrong with them" (individual walking interview, FG6). This combination of a predominantly open membership combined with group norms that encouraged informal engagement between different members meant that people were recognised and valued as 'full' members, irrespective of the presence or absence of dementia:

Everybody has become good friends: we are all very aware of individuals' abilities within the group, so it is not just us-you are looking out for each other. Some of you come with your partners, but you do not stay with your partners. You maybe use it as an opportunity not to and walk with other people. It is not just us. You all do, as I say, look out for each other now. (FG2)

This sense of reciprocal social support simultaneously facilitated and was facilitated by a practice of open and frank communication between those walking about the challenges they were facing in their lives. While participants reported feeling respected and supported by those they confided in, irreverent humour and good-natured 'banter' often became a key feature of such discussions when they occurred. This humour was observed during fieldwork and discussed at length by participants, on a range of subjects from situational humour to more sensitive subjects such as ill-health, dementia and death. That these conversations could occur in a way that allowed walkers to express their feelings and experiences without becoming unnecessarily maudlin was discussed as a function of their relationships. One person, a former carer of a person with dementia who had passed away, captured this when discussing feeling guilty over choosing to move the person they were caring for into a care home, saying:

I lost my wife in the last couple of years...and I have found this has been absolutely brilliant to come to something like this, meet all these people, and get out the house. It gives you something to focus on instead of sitting at home feeling sorry for myself. It is of great benefit to me. (FG3) 
That such support was available at a point where an individual's social role, identity, and networks may be particularly vulnerable highlights the value of walking groups as a locus of support, especially for those who lived alone. Being part of such a group often offered an opportunity to connect organically with others who had shared a similar experience, or who expected they might do so in the future. This allowed a greater level of understanding between members and fostered enabling (rather than disabling) practices. The value of such a resource is particularly clear for those with dementia, for whom the journey is often uncertain and who often face a reduction in social support and a 'shrinking world.' By attending the walks consistently over time, group members were able to form their own sense of community, as one focus group participant remarked:

It is part of the group-it is part of belonging. When you come to our group, we end up belonging to one another. You are brothers and sisters in this group because we all talk to one another and we all have fun together in whatever way we can-just being there for each other. (FG6)

The mechanisms for this belonging and inclusivity presented in a number of ways. Friendships emerging from the walking group frequently extended beyond organised sessions, with participants reporting attending additional social activities, such as going to the pub, attending exercise classes or taking part in cultural activities separate from the walking group. Walking group members used the time before the walk began to 'catch up,' a process that allowed those who walked at different speeds or on different routes to maintain relationships. Once the walk had begun, different group members were observed (and later reflected upon) moving between clusters or partners as their energy or the terrain changed. Some of the most influential interactions occurred after the walk, as each group was arranged to finish at a café where participants could sit and socialise informally. Rather than a supplementary aspect to a walking group otherwise focused on physical health, these café stops served to support social health, cementing the social bonds grown during walks.

\subsection{Reciprocity and Looking Out for Each Other: Creating a Safe and Secure Social Environment}

A secondary theme at once distinct from and contingent on the theme of social inclusion and participation was that groups created a safe and secure social environment where participants engaged in meaningful activities supportive of their physical, mental, and social health. The importance of the walking group as a formal activity facilitated by volunteers and designated walk leaders and populated by supportive and accepting groups of individuals was reflected in individual interviews and focus groups. One person with dementia, for example, gestured to the industrial estate the group was passing, expressing to the researcher that they would not come there themselves: "I would never get out of here. I would need to run into one of those offices and shout for help" (individual walking interview, FG3).

Despite explicitly discussing the role of the walking group in addressing an aspect of her dementia (challenges with disorientation and wayfinding), this person did not view this as a function of the dementiafriendliness of the group, but its facilitative nature overall, later noting:

[The group] gets the people out-people that cannot get out on their own-and somebody is looking after them while they are out. When I am away on the likes of these things, he [my husband] does not bother about me because I am with people that are looking after me. I am getting looked after while I am there. (individual walking interview, FG3)

Discussions of safety and security were as important to walkers with and without dementia as they were to walk leaders. One volunteer related the following experience where they had supported a couple who were anxious about entering a new social space and new activity. After receiving encouragement and support, both demonstrated greater levels of social health, with the walk leader reporting:

[The person with dementia] never used to speak; now [she] can speak for Scotland. She came along in the very first walk and he [her husband] was very anxious - he wouldn't leave her. He did and literally after the third walk, he said that he was fine with these people-you go with your group. (FG3)

Not only did the carer experience and accept the walking group as a safe space where both he and his wife could participate, but he recognised the group as somewhere where his wife could experience belonging and social integration as she walked with 'her' group. Such reflections are indicative of a wider ethos of dementia-inclusiveness, and as such supported people with dementia to connect and remain connected to others within their community. This practice of considering the needs of people with dementia alongside more familiar concerns around physical mobility, access and risk assessment by those who had adopted this inclusive ethos was carried forward into other spaces and activities where people with dementia might encounter barriers to their participation, as one health walk coordinator noted:

I think for us [becoming dementia-friendly] brought dementia into focus more, and, actually, it made...us think about our other activities because we have not only walks, we have other different activities...cropping up from our buddy swimming programme [to] our indoor curling. (FG7) 
Participants also valued the walking groups as a place where family carers and the person they cared for could spend some time apart, gaining some respite from each other during walks as they spoke to and accompanied other people during the walk itself. That groups provided opportunities for mixed abilities (such as by offering different length walks depending on a person's mobility), as well as support from other group members who attended walks, meant carers could feel comfortable that their partner would be looked after by others, while they could spend some time with other people.

As such, people with dementia and their carers could gain different things from walking groups, from simply being in the outdoors with other people for people with dementia, while carers also gained some respite from the demands of care. Groups therefore became a potential catalyst for people with dementia to develop new social relationships and networks, while supporting them to be active participants in their local communities.

\subsection{Accessing the Outdoors: Promoting Agency and Capacity through Physical Activities}

Having discussed the social benefits of walks and the factors that ensure walkers experienced the activity as safe and supportive, this section highlights how walking groups functioned as part of a wider initiative to support attendees to take proactive steps to maintain their health. Such an extension represents not only a shift in analytic theme, but what was often an evolution of discussions that occurred in focus groups with walkers and walk leaders, where walking was discussed as both a proactive and reactive response to health concerns. Indeed, one health walk coordinator highlighted the impact of the wider cultural push towards social health and integration on their ability to recruit new members, with different stakeholders recognising the value of walking groups as a resource:

With the whole health and social care agenda going on, we are starting to make more contacts and more inroads into services, who, traditionally, have just done it their way and [with] medication. (FG7)

This positioning of walking groups as a valuable and valid method of promoting and maintaining health was reflected by other participants who emphasised that, while the group might be dementia-inclusive, it was not itself a 'dementia group.' This was epitomised by one participant who explained:

We are here to get fit. It is nothing to do with dementia as far as I am concerned....We have noticed that everybody has got fitter over the year because we are actually going round the courses a lot quicker. [We] have been talking about trying to extend them a bit longer for the Thursday walk. There is a big difference in your health. (FG3)
Such sentiments were shared across groups, even within the group that had been explicitly arranged to support people with dementia and carers rather than the wider community. Walking, they argued, was an activity that could be made accessible for people at various stages of their dementia journey, but that did not itself mean the walking group needed to pivot to focus on dementia. Instead, walking groups functioned as spaces where the challenges faced by people with dementia were validated and respected alongside the challenges faced by other members rather than in isolation from them. Even those who did not explicitly link walking with fitness acknowledged the physical gains they received. Such activities could reassert confidence, agency and capacity and were socially-situated; exercise was more enjoyable when taking part with other people, highlighting the holistic benefits of integrating physical and social health in one activity: "If you are on the bike or if you are on the treadmill, it is just you, whereas when you are out on the walk, you have all the others" (FG1).

This focus on enabling continued participation via walking as a healthy and meaningful activity reinforced a broader commitment to and acceptance of adaptation and enablement for all members irrespective of formal diagnosis. The following example was provided in a group that regularly accessed natural spaces and uneven walkways:

Some people...were unsteady so [a member] advised that somebody try using a pole, which I think [someone] offered to do and she benefited from that. The next time we were out another one of the members...was a bit unsteady and I asked him if he would like to try a pole. He said he thought it would help. So, we thought we would get some for the group and just leave them here so that if anybody is having a bad day or wants to try one, they are here. (FG2)

First the pragmatic recognition of poor balance shows up as an issue of a body-in-space that might be effectively addressed with a mobility aid, and the willingness of a group member to test out this approach. This enabling focus had a positive impact on other group members who were encouraged, without judgement, to try similar practices. These changes were framed as being facilitative of continued engagement with walking rather than as responses to dementia per se, opening up further avenues for support to others for whom such steps are useful.

The reassurance that, ultimately, walking groups were a health activity with a valued and valuable social component encouraged members to recognise that they were "not going for a race" (FG2) ensuring that members could seek support and adapt to changes in their abilities without judgement or exclusion. The walking groups supported social health by enabling a person to maintain capacities, fulfilling their potential by promoting physical fitness and thereby their identity as active, capable and autonomous individuals. 


\section{Discussion}

Providing much-needed insight into how national initiatives can be enacted at the local level to support the social health of people with dementia as both individuals and citizens in their own right, this analysis offers new insights regarding walking groups as a 'dementiaenabling' activity that supports social health by enabling the inclusion and integration of people with dementia within the wider community. While literature on 'dementia-friendly' communities has tended to focus narrowly on the built environment, we extend the notion of 'dementia-enabling' environments to report on how walking initiatives can support people living with dementia to remain connected to their communities via valued outdoor spaces. This study advances our understanding of how a dementia-inclusive outdoor initiative supports continued social participation for people living with dementia. A supportive, inclusive walking group that integrates people with dementia in their local community has the potential to promote continued social participation by providing a place where new social relationships can flourish despite a person's dementia. Specific benefits were in providing people with dementia with a relatively safe and secure place to socialise and interact meaningfully with others, while providing carers with opportunities for a brief period of respite from their caregiving role, simultaneously exerting the person with dementia's autonomy while supporting them within the group. In doing so, walking groups supported the continued social health of people with dementia and carers by providing an opportunity to maintain existing and create new interdependencies despite the 'shrinking world' commonly associated with the condition.

The concept of social health, as applied to dementia, provides a useful tool through which to capture the health and related benefits of social 'interventions' such as 'dementia-friendly' walking groups (Dröes et al., 2017). Far from Swaffer's (2015) experience of prescribed disengagement, walking groups promote social health by giving attendees with dementia the opportunity to engage in physical activities which sustain physical health, while both maintaining existing and building new meaningful relationships within the physical and social spaces of their communities, thereby supporting a person's remaining capacities and reinforcing normalcy in their lives. The walking group initiatives gave attendees the opportunity to maintain a coherent self-identity, to participate and contribute to their communities, and continue to both receive and give support within the social relationships existing within the initiatives (Dröes et al., 2017). Such benefits were also strongly associated with groups as being inclusive of people with dementia as part of their wider communities. When compared to more traditional service-led models where support for people with dementia can be segregated from the rest of their communities (e.g., residential care), or which may be labelled as activities exclusively or predominantly for people with dementia (e.g., dementia cafés or support groups), such activities promoted continued social engagement and civic participation, with the expanded horizon experienced by attendees made possible by the integrative and inclusive, community-focused approach adopted within the initiatives. Few people with dementia spoke of walks as being explicitly targeted at them, nor did any participants speak of joining walks specifically because of their dementia. The general feeling was that walking groups created a place where a person's dementia did not pose a barrier to their participation, rather than being a space exclusively for people with dementia, or where dementia was the reason for their presence. Walking groups were dementia 'supporting' spaces, without being dementia exclusive spaces. Such groups thereby supported attendees to fulfil their potential as participating members of their communities, rather than as a person diagnosed with dementia; as a patient, 'sufferer' or individual defined through their needs (Vernooij-Dassen, Moniz-Cook, \& Jeon, 2018).

A key characteristic of five of the six groups were that walks were not limited to people with dementia and their carers, but supported people living with dementia to engage with the wider population of individuals attending walks. This inclusive basis, in which activities were not framed as being specifically dementia-related activities, became one of the key facilitators of continued social health among attendees. Groups were generally perceived as being inclusive and encouraging to all people, with 'dementia-friendliness' being a secondary benefit of the inclusive atmosphere attached to groups. Perhaps counter-intuitively, and unlike Phinney et al.'s (2016) example of a walking group designed exclusively for people with dementia but which discouraged people from discussing their dementia, the inclusive and integrated model supported attendees with dementia to find a place for themselves socially as people with dementia, but within an inclusive rather than exclusive social milieu. Dementia was a presence in the walks, but the reciprocal nature of walking groups, in which volunteers and other walkers supported each other regardless of whether a person was affected by dementia or another long-term condition, engendered the community affiliations attached to each group. Those who were most vulnerable to social isolation, for example those bereaved or living alone, found groups to be particularly valuable. Extending Odzakovic et al.'s (2018) conclusion that walking in one's neighbourhood can support social relationships, this analysis establishes that a structured and inclusive walking group goes further by facilitating continued community participation among people living with dementia, especially those isolated in their communities.

The study illustrates the complex interdependencies linked to social participation which can be strengthened through links to the outdoors and the natural environment. The potential benefits of access to nature has been underplayed in the literature on 'dementia-friendly' communities, which has tended to focus narrowly on the 
built environment. In this article, we extend the notion of 'dementia-inclusive' environments to demonstrate the benefits of accessing nature: 'blue' or 'green' spaces in the environment. With the structure of groups supporting capacity and abilities as opposed to deficits and loss, walking within these environments was framed as an activity that built fitness, promoting agency and active participation within a social and physical space that encouraged people to feel secure, whilst also having fun. The practice of walking could help re-instil pride in the accomplishment of embodied health, which dementia may erode. The focus on broad physical health as opposed to dementia was helpful as a frame of referenceacknowledging and recognising the condition yet not defining membership of the group as based on their dementia. Socially, walking groups supported people to fulfil their potential by focusing on their abilities, maintaining autonomy and supporting both social networks and meaningful activities, defined by Huber et al. (2011) and Dröes et al. (2017) as core dimensions of social health.

\section{Conclusion}

Our findings provide new insight into how social initiatives and interventions embedded in communities, and which facilitate continued participation of people with dementia with other people within these communities, can improve the social health of people living with dementia (Vernooij-Dassen et al., 2018). Inclusive walking groups such as those discussed here provide valuable opportunities for people living with dementia to access outdoor spaces and engage in meaningful social interactions. In doing so, this enables people to fulfil their potential and obligations, maintain a degree of autonomy and independence despite their illness, and continue to be socially involved within their local communities (Dröes et al., 2017). From the analysis conducted here, the place where walks took place was important, for example in terms of supporting an individual's ability to access both green spaces, as well as maintaining a connection with the local communities in which they dwelled, in some cases for most or all of their lives. The adoption of an inclusive community-based approach in which social interventions for people with dementia are socially situated and embedded within their local communities is illustrated as an important element of such social interventions in promoting social health. Walking groups gave people the opportunity to mix and socially participate in a supportive environment, but which was not labelled as being in a physical or social space seen as exclusively for those with dementia. In doing so, such interventions reinforced a sense of normalcy, rather than of a dementiabased 'exceptionalism' which can be a feature of many dementia-specific interventions. Our findings highlight the value of viewing 'dementia-friendliness' in relation to the wider inclusiveness of initiatives that might involve people living with dementia with their wider communities, rather than building distinct but potentially ghetto- ised 'dementia-friendly' spaces within communities. This demonstrates the value of designing support for people with dementia that integrates them in local communities. This standpoint is reinforced by wider evaluations of 'dementia-friendly' communities, which argue for the normalisation of people with dementia in services where possible, rather than creating dementia 'exclusive' services or spaces (Buckner et al., 2019). Future initiatives could benefit from focusing on inclusivity within wider communities rather than focusing on interventions specifically and exclusively for people with dementia. To this end, we suggest that the term 'dementia-inclusive' may be a more appropriate term for initiatives to adopt when compared to the phrase 'dementia-friendly' so that such initiatives can act as catalysts for growing levels of community participation.

\section{Acknowledgments}

We gratefully acknowledge the participants, volunteers and staff in the walking groups who generously gave their time and support for this project. Many thanks to Richard Ward and Alison Bowes who provided comments on earlier drafts of this article.

\section{Conflict of Interests}

The authors declare no conflict of interests.

\section{References}

Alzheimer's Disease International. (2019). Global dementia charter: I can live well with dementia. London: Alzheimer's Disease International.

Bartlett, R. (2016). Scanning the conceptual horizons of citizenship. Dementia, 15(3), 453-461.

Blake, M., \& Mitchell, G. (2016). Horticultural therapy in dementia care: A literature review. Nursing Standard, 30(21), 41-47.

Braun, V., \& Clarke, V. (2006). Using thematic analysis in psychology. Qualitative Research in Psychology, 3(2), 77-101.

Buckner, S., Darlington, N., Buswell, M., Mathie, E., Arthur, A., Lafortune, L., . . Goodman, C. (2019). Dementia friendly communities in England: A scoping study. International Journal of Geriatric Psychiatry, 34(8). https://doi.org/10.1002/gps.5123

Clark, A., \& Emmel, N. (2010). Using walking interviews. Morgan Centre for Research into Everyday Lives. Retrieved from https://www.socialsciences. manchester.ac.uk/morgan-centre/research/ resources/toolkits/toolkit-13

Department of Health. (2015). Prime Minister's challenge on dementia 2020: Implementation plan. London: UK Government.

Dewing, J. (2007). Participatory research: A method for process consent with persons who have dementia. Dementia, 6(1), 11-25. 
Dröes, R., Chattat, R., Diaz, A., Gove, D., Graff, M., Murphy, K., . . IINTERDEM Social Health Taskforce. (2017). Social health and dementia: A European consensus on the operationalization of the concept and directions for research and practice. Aging \& Mental Health, 21(1), 4-17.

Duggan, S., Blackman, T., Martyr, A., \& van Schaik, P. (2008). The impact of early dementia on outdoor life: A 'shrinking world'? Dementia, 7(2), 191-204.

Genoe, M. (2010). Leisure as resistance within the context of dementia. Leisure Studies, 29(3), 303-320.

Gibson, A., Ramsden, N., Tomlinson, R., \& Jones, C. (2017). Woodland wellbeing: A pilot for people with dementia. Working with Older People, 21(3), 178-185.

Gonzalez, M., \& Kirkevold, M. (2013). Benefits of sensory garden and horticultural activities in dementia care: A modified scoping review. Journal of Clinical Nursing, 23(19/20), 2698-2715.

Greasley-Adams, C., Pengelly, R., Aitken, J., Berry, P.-Y., Cameron, D., Chalmers, T., . . . 6 Community Researchers Who Remain Anonymous. (2019). A good life in later years. In Co-production in action no. 2 (pp. 15-26). London: National Institute for Health Research. Retrieved from https://www.invo.org. uk/posttypepublication/co-production-in-actionnumber-two

Greasley-Adams, C., Robertson, J., Gibson, G., McCall, V., Aitken, J., Berry, P.-Y., . . . 6 Community Researchers Who Remain Anonymous. (2017). A good life in later years: A co-produced research project (Final Report). Glasgow: Life Changes Trust. Retrieved from https://www.lifechangestrust.org.uk/sites/ default/files/publication/files/Good\%20Life\%20in\% 20Later\%20Years\%20FINAL\%20NOV\%2017.pdf

Hendriks, I., van Vliet, D., Gerritsen, D., \& Dröes, R. (2016). Nature and dementia: Development of a person-centred approach. International Psychogeriatrics, 28(9), 1455-1470.

Huber, M., Knottnerus, A., Green, L., van der Horst, H., Jadad, A., Kromhout, D., . . . Smid, H. (2011). How should we define health? BMJ, 343. https://doi.org/ 10.1136/bmj.d4163

Noone, S., Innes, A., Kelly, F., \& Mayers, A. (2017). The nourishing soil of the soul: The role of horticultural therapy in promoting well-being in communitydwelling people with dementia. Dementia, 16(7), 897-910.

Odzakovic, E., Hellström, I., Ward, R., \& Kullberg, A. (2018). 'Overjoyed that I can go outside': Using walk- ing interviews to learn about the lived experience and meaning of neighbourhood for people living with dementia. Dementia. Advance online publication. https://doi.org/10.1177\%2F1471301218817453

Ottmann, G., Laragy, C., Allen, J., \& Feldman, P. (2011). Coproduction in practice: Participatory action research to develop a model of community aged care. Systemic Practice \& Action Research, 24(5), 413-427.

Phinney, A., Kelson, E., Baumbusch, J., O’Connor, D., \& Purves, B. (2016). Walking in the neighbourhood: Performing social citizenship in dementia. Dementia, 15(3), 381-394.

Prince, M., Knapp, M., Guerchet, M., McCrone, P., Prina, M., Comas-Herrera, A., .. . Salimkumar, D. (2014). Dementia UK: Update. London: Alzheimer's Society.

Shakespeare, T., Zeilig, H., \& Mittler, P. (2019). Rights in mind: Thinking differently about dementia and disability. Dementia, 18(3), 1075-1088.

Swaffer, K. (2015). Dementia and prescribed disengagement. Dementia, 14(1), 3-6.

Vaughn, L. M., Whetstone, C., Boards, A., Busch, M. D., Magnusson, M., \& Määttä, S. (2018). Partnering with insiders: A review of peer models across communityengaged research, education and social care. Health \& Social Care in the Community, 26(6), 769-786.

Vernooij-Dassen, M., \& Jeon, Y. (2016). Social health and dementia: The power of human capabilities. International Psychogeriatrics, 28(5), 701-703.

Vernooij-Dassen, M., Moniz-Cook, E., \& Jeon, Y. (2018). Social health in dementia care: Harnessing an applied research agenda. International Psychogeriatrics, 30(6), 775-776.

Ward, R., \& Campbell, S. (2013). Mixing methods to explore appearance in dementia care. Dementia, 12(3), 337-347.

Ward, R., Clark, A., Campbell, S., Graham, B., Kullberg, A., Manji, K., . . . Keady, J. (2018). The lived neighbourhood: Understanding how people with dementia engage with their local environment. International Psychogeriatrics, 30(6), 867-880.

Wild, A., Clelland, D., Whitelaw, S., Fraser, S., \& Clark, D. (2018). Mapping the 'housing with care' concept with stakeholders: Insights from a UK case study. Journal of Integrated Care, 26(4), 257-266.

Wright, T. (2014). Reconceptualising dementia friendly communities. Diversity \& Equality in Health \& Care, 11(3), 282-283.

Yuill, C., Crinson, I., \& Duncan, E. (2010). Key concepts in health studies. London: Sage.

\section{About the Authors}

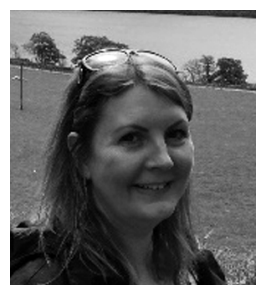

Jane M. Robertson is Programme Director for Dementia Studies at the University of Stirling. Her central interest is contributing knowledge and innovation that improves the quality of life and wellbeing of older people, people with dementia and carers through co-produced research. She is currently investigating peer support for people with dementia and carers, inclusive and assisted living in supported housing schemes, creative arts-based interventions in continuing care settings, and evaluating the impact of services and support for people affected by dementia. 


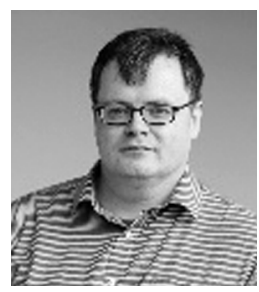

Grant Gibson is Lecturer in Dementia Studies in the Faculty of Social Sciences at the University of Stirling. His research interests include the design and implementation of assistive technologies, social and community interventions in dementia care and the use of participatory research methodologies with people affected by dementia. His research is currently exploring the use of physical activity interventions in care homes and the community, and use of assistive technologies within supported housing schemes.

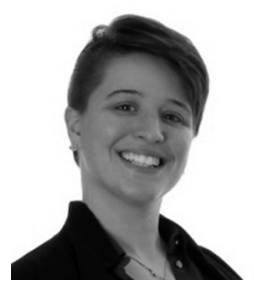

Catherine Pemble holds an MSc in Psychological Research Methods and a PhD in Dementia Studies from the University of Stirling. Her work focuses on using qualitative methods to understand the experiences of vulnerable people and how they manage their relationships with the people and services that are important to them.

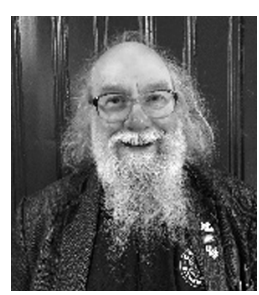

Rog Harrison retired after 38 years as a Civil Servant with the Inland Revenue/HMRC and trained as a Volunteer Community Researcher with the Faculty of Social Sciences at the University of Stirling in 2016. He then worked with the University of Stirling on the "A Good Life in Later Years" project and the evaluation of dementia-friendly walking for "Paths for All." He is currently working with the University of Stirling on an evaluation of the Life Changes Trust's Peer Support Projects.

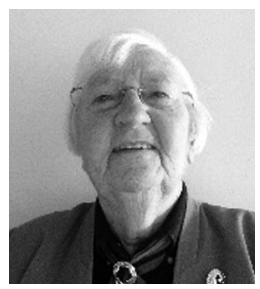

Kim Strachan worked for 40 years in the financial sector and served in the WRNS working with radar. She moved into voluntary work with the National Health Service and Ambulance Service immediately on retirement. Kim has worked as a Volunteer Community Researcher in the Faculty of Social Sciences at the University of Stirling since 2016, with a keen interest in developing knowledge about ageing and dementia. She describes her experience as informative, encouraging and uplifting.

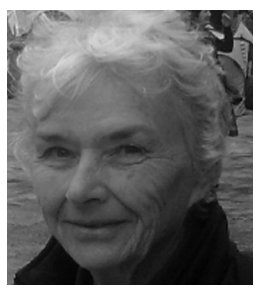

Sheila Thorburn joined the Faculty of Social Sciences, University of Stirling, as a Volunteer Community Researcher in 2016. Working, initially, on the "A Good Life in Later Years" project, experience was gained in collecting, refining and analysing qualitative data related to ageing and wellbeing. Since that time this experience has been used in other co-produced projects at the University of Stirling including the evaluation of dementia-friendly walking for "Paths for All." 